\title{
Self-Diffusion at a Melting Surface Observed by He Scattering
}

\author{
J. W. M. Frenken, J. P. Toennies, and Ch. Wöll \\ Max Planck Institut für Strömungsforschung, 3400 Göttingen, Federal Republic of Germany \\ (Received 14 October 1987)
}

\begin{abstract}
Self-diffusion at surfaces can be studied with quasielastic scattering of low-energy $\mathrm{He}$ atoms. This is demonstrated for the $\mathrm{Pb}(110)$ surface close to the melting point, $T_{m}^{\mathrm{Pb}}=600.7 \mathrm{~K}$. From the width of the quasielastic energy distribution of scattered $\mathrm{He}$ atoms it is inferred that at $T \geqq 450 \mathrm{~K}$ the surface atoms are anomalously mobile. At $T \geqq 550 \mathrm{~K}$, surface mobilities are found to exceed the bulk-liquid value. These results complement recent ion-scattering measurements on surface disorder of $\mathrm{Pb}(110)$.

PACS numbers: $64.70 . \mathrm{Dv}, 66.30 . \mathrm{Fq}, 68.90 .+\mathrm{g}$
\end{abstract}

The quasielastic scattering of thermal-energy neutrons $^{1}$ is a well-established technique to measure selfdiffusion on an atomic scale in the bulk of threedimensional systems. Self-diffusion at the surface of a solid can be studied at sufficiently low temperatures with experimental methods like field-ion microscopy ${ }^{2}$ and the field-emission current-fluctuation technique. ${ }^{3}$ At higher temperatures, e.g., $T>0.5 T_{m}$, self-diffusion on the surface of three-dimensional solids has so far only been investigated with macroscopic-scale techniques (e.g., mass-transfer, tracer diffusion). ${ }^{4,5}$

In this Letter, we present the first quasielasticscattering measurements performed with thermal-energy He atoms. It is demonstrated that, with sufficient energy resolution, this novel application of atom-surface scattering can be used to probe directly the intrinsic lateral diffusion at the surface of a three-dimensional metal crystal. We have used this new technique to study surface melting of $\mathrm{Pb}(110)$.

Among phase transitions at surfaces of crystals, surface melting is currently receiving special attention in view of its possible role in initiating bulk melting. ${ }^{6}$ Surface melting is a continuous and reversible process, which takes place below the bulk melting point $T_{m}$, and involves a positional disordering of the crystal lattice in the surface region. ${ }^{6-9}$ As the temperature approaches $T_{m}$, the thickness of the disordered region diverges. The melt depth shows a strong dependence on crystal face, the most densely packed faces remaining stable up to $T_{m}$, and the most open surfaces exhibiting the strongest surface-melting effect. ${ }^{8}$

Up to now, experiments on surface melting have mainly been focused on the loss of crystalline order at the surface. None of the studies have really distinguished between a liquidlike surface layer and a strongly disordered solid surface region (i.e., microcrystalline or glassy). In order to decide whether or not the disordered surface layer can be correctly described as a "quasiliquid," knowledge is required of the atomic mobility within this layer. Molecular-dynamics calculations predict liquidlike diffusivities in the outermost atomic layer at temperatures close to $T_{m} \cdot{ }^{10,11}$ Experimentally, atomic-scale information on surface diffusivity just below $T_{m}$ has so far only been obtained for thin adsorbed methane films with the quasielastic scattering of thermal-energy neutrons. ${ }^{12}$ The quasielastic He-scattering measurements reported here show that $\mathrm{Pb}$ atoms at the $\mathrm{Pb}(110)$ surface attain liquidlike mobilities at temperatures as much as $50 \mathrm{~K}$ below $T_{m}\left(T_{m}^{\mathrm{Pb}}=600.7 \mathrm{~K}\right)$.

The $\mathrm{Pb}$ specimen used in this study was spark cut from a single-crystal $\mathrm{Pb}$ ingot, chemically polished, and subsequently cleaned in ultrahigh vacuum by cycles of Ar-ion sputtering and thermal annealing, as described in Ref. 7. Crystal temperatures up to $T_{m}$ were obtained by radiative heating of the reverse side of the Mo sample holder. The temperature was monitored with an infrared pyrometer (Ircon model 6000) and a Pt resistance thermometer. Surface cleanliness and crystalline order were checked with Auger-electron spectroscopy and $\mathrm{He}$-atom diffraction. The He-scattering experiments were performed with supersonic He nozzle beams with unusually low energies around 2.2 or $6.5 \mathrm{meV}$. Scattered $\mathrm{He}$ atoms were detected at a fixed scattering angle of $90^{\circ}$ with respect to the incident beam, as a function of energy, with time-of-flight analysis. The energy resolution, FWHM, of the complete system, including both the nozzle beam and the detector, amounted to $\simeq 80$ and $\simeq 170$ $\mu \mathrm{eV}$ at the beam energies of 2.2 and $6.5 \mathrm{meV}$, respectively, as determined from measurements of the (purely elastic) specular beam.

The principle of the He-scattering measurements reported here is similar to that of quasielastic neutron scattering. ${ }^{1}$ When a beam of $\mathrm{He}$ atoms is reflected from a fluid surface, the elastic peak in the energy distribution of diffusely scattered $\mathrm{He}$ atoms is, in fact, weakly inelastic. The broadening of the reflected energy distribution with respect to the incident energy distribution is brought about by small energy transfers related to the diffusive motion of the surface atoms, similarly to Doppler broadening. The theory of "quasielastic" scattering, which was originally developed for thermalenergy neutron-scattering experiments from bulk specimens, ${ }^{13}$ predicts for random continuous diffusion a Lorentzian energy profile with a FWHM of

$$
\Delta E=2 \hbar D \Delta k^{2}
$$




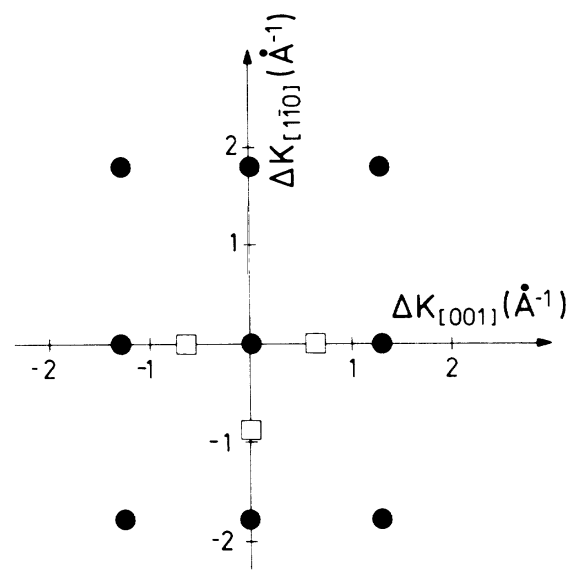

FIG. 1. Reciprocal-space diagram of the $\mathrm{Pb}(110)$ surface, showing the surface reciprocal lattice (circles) and the $\Delta \mathbf{K}$ locations where the quasielastic scattering measurements were performed (squares).

where $D$ is the diffusion coefficient and $\hbar \Delta k$ the magnitude of the momentum transfer. For thermal-energy He-atom scattering from a two-dimensional fluid adsorbate, Levi, Spadacini, and Tommei have shown that the expected broadening amounts to

$$
\Delta E=2 \hbar D_{s} \Delta K^{2}
$$

Here, $\hbar \Delta K$ is the magnitude of the component of the momentum transfer parallel to the surface and $D_{s}$ is the intrinsic surface diffusion coefficient in the direction of $\Delta \mathbf{K}^{14}$

Figure 1 shows a reciprocal-space diagram of the $\mathrm{Pb}(110)$ surface. The quasielastic energy distributions reported in this Letter were measured at the Brillouinzone boundary positions marked by the squares, along the [001] and [1 $\overline{1} 0]$ surface azimuths.

In Fig. 2 a selection of measured energy spectra is displayed of $\mathrm{He}$ atoms scattered from $\mathrm{Pb}(110)$ at crystal temperatures of 446,544 , and $551 \mathrm{~K}$, with a beam energy of $6.5 \mathrm{meV}$ and an incident angle of $37.5^{\circ}$ with respect to the surface normal, corresponding to $\Delta \mathbf{K}$ $=0.64 \AA^{-1}$ along the [001] azimuth. The measurements have been corrected by subtraction of a smoothly varying inelastic background. The dashed Gauss curves illustrate the instrumental energy resolution of $\Delta E_{\text {res }}=163 \mu \mathrm{eV}$ in Fig. 2, which could be determined from measurements performed either at room temperature or at $\Delta \mathbf{K}=0$. The full curves are Gauss fits to the data. ${ }^{15}$ Figure 2 clearly demonstrates the quasielastic energy broadening with increasing temperature. Before discussing the energy broadening, we mention that the intensities of the specular peak and the diffraction peaks, as well as the quasielastic signal, were found to decrease strongly at temperatures above $\simeq 500 \mathrm{~K}$. This is probably caused by strong anharmonicity of the surface vibrations at these temperatures, as was proposed also in

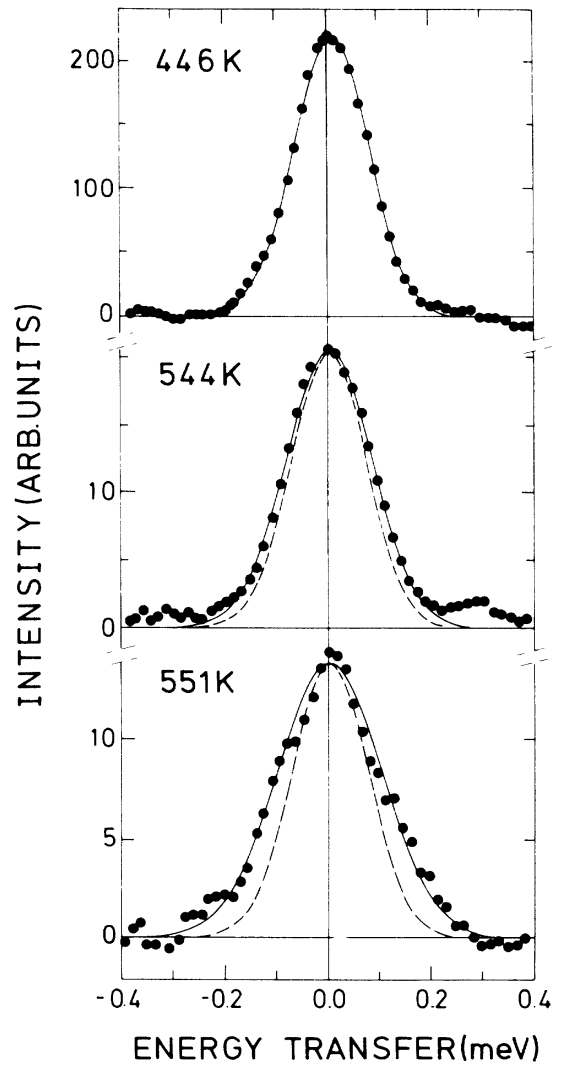

FIG. 2. Energy distributions of $\mathrm{He}$ atoms scattered from a $\mathrm{Pb}$ (110) surface, at three crystal temperatures, for $\Delta \mathbf{K}=0.64$ $\AA^{-1}$ along the [001] surface azimuth. The most probable beam energy is $6.5 \mathrm{meV}$. The dashed curves show the experimental resolution of $163 \mu \mathrm{eV}$. The full curves are Gauss fits to the data.

the case of a similar loss of elastic intensity for $\mathrm{Cu}(100){ }^{16} \mathrm{~A}$ further account of this will be given in a later publication. ${ }^{17}$ As a result of the drop in elastic and quasielastic intensity, the quasielastic peak became too small at temperatures above $=570 \mathrm{~K}$ to allow for a determination of its energy width in the present experiment.

In Fig. 3 energy widths $\Delta E$ are shown as a function of crystal temperature, which were obtained by correcting the measured widths $\Delta E_{\exp }$ for the resolution $\Delta E_{\text {res }}$ with ${ }^{15}$

$$
\Delta E=\left[\left(\Delta E_{\text {exp }}\right)^{2}-\left(\Delta E_{\text {res }}\right)^{2}\right]^{1 / 2} .
$$

The top panel of Fig. 3 is for $\Delta \mathbf{K}= \pm 0.64 \AA^{-1}$ along the [001] surface direction; the bottom panel is for $\Delta \mathbf{K}=0.90 \AA^{-1}$ along [1 $\left.1 \overline{1} 0\right]$. The different symbols in Fig. 3 correspond to different beam energies. The perpendicular momentum transfer changes, between 2.2 and $6.5 \mathrm{meV}$, by almost a factor 2, for a given parallel momentum transfer. However, the observed $\Delta E$ values for the two different incident energies are, to within experimental accuracy, equal. This shows that either the 


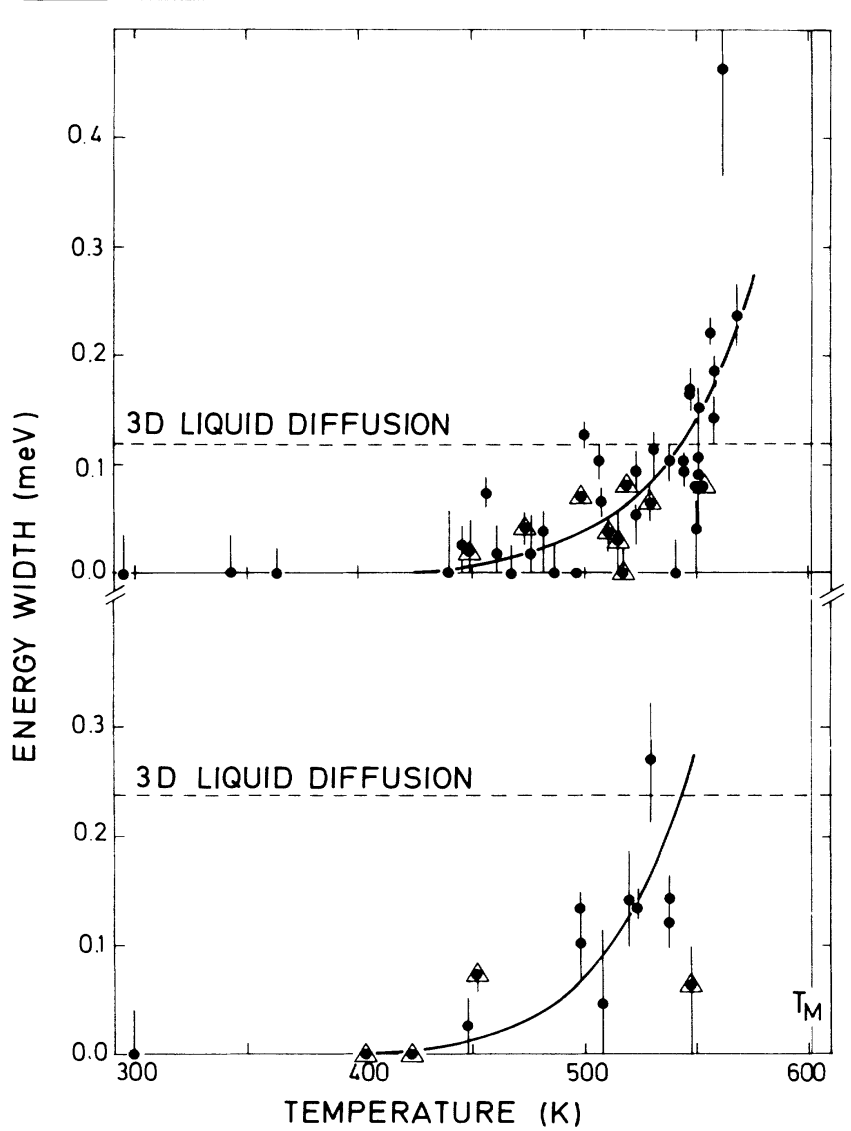

FIG. 3. Top panel: Temperature dependence of the energy width of the quasielastic peak in the energy distribution of He atoms scattered from $\mathrm{Pb}(110)$ with $\Delta \mathbf{K}= \pm 0.64 \AA^{-1}$ along [001] for initial beam energies of 2.2 (triangles) and $6.5 \mathrm{meV}$ (circles). The dashed line shows the energy width expected for bulk liquid $\mathrm{Pb}$. The full curve is discussed in the text. Bottom panel: Same as top panel, for $\Delta \mathbf{K}=0.90 \AA^{-1}$ along the [1ī0] surface direction.

quasielastic He-scattering measurements are predominantly sensitive to the lateral diffusive motion, or the diffusion coefficient in the perpendicular direction is much smaller than the lateral diffusion coefficient. In both cases, it justifies the use of a relation between $\Delta E$ and $\Delta K$ only, as was assumed in Eq. (2).

The dashed lines in Fig. 3 indicate the energy widths expected from Eq. (2) for the bulk diffusion coefficient $D_{b}=2.2 \times 10^{-5} \mathrm{~cm}^{2} \mathrm{~s}^{-1}$ of liquid $\mathrm{Pb}$ just above $T_{m} .{ }^{18}$ At the surface this value is reached already at $\simeq 50 \mathrm{~K}$ below $T_{m}$. The full curves in Fig. 3 represent energy widths calculated with Eq. (2) for a surface self-diffusion coefficient which varies with temperature as

$$
D_{s}(T)=D_{0} \exp \left(-Q_{s} / k_{\mathrm{B}} T\right) \text {, }
$$

with $D_{0}=26 \mathrm{~cm}^{2} \mathrm{~s}^{-1}$ and $Q_{s}=0.65 \mathrm{eV}, k_{\mathrm{B}}$ being the Boltzmann constant. The energy widths of Fig. 3 are consistent with such a behavior. Because of the large scatter in the measured energy widths in Fig. 3, the above choice of the activation energy $Q_{s}$ is estimated to be correct only to within $\pm 0.2 \mathrm{eV}$. Although the [001] and [1 10$]$ directions are strongly inequivalent at the (110) surface of an fcc crystal, being perpendicular and parallel to the [110] surface channels, respectively, the diffusion coefficients along these two directions are apparently equal within the present experimental accuracy. This might seem surprising, but surface self-diffusion studies at low temperatures have revealed that a "knockout" mechanism can lower the activation energy for cross-channel diffusion appreciably. ${ }^{3,19}$ In addition, high-temperature mass-transfer experiments have been interpreted in terms of the existence of a twodimensional gaslike diffusive state at the surface, which would be relatively insensitive to the surface structure. ${ }^{4}$

It is instructive to compare the diffusion coefficients and activation energy found here for the surface with those known for bulk-solid and -liquid $\mathrm{Pb}$. Close to $T_{m}$ the solid and liquid diffusion coefficients are $4.5 \times 10^{-10}$ and $2.2 \times 10^{-5} \mathrm{~cm}^{2} \mathrm{~s}^{-1}$, respectively, ${ }^{18,20}$ while extrapolation of Eq. (2) to $T_{m}$ gives a surface value of 9.2 $\times 10^{-5} \mathrm{~cm}^{2} \mathrm{~s}^{-1}$. Molecular-dynamics calculations for Lennard-Jones systems reveal that surface diffusion coefficients are higher than bulk-liquid diffusion coefficients for temperatures close to melting. ${ }^{10,11}$ This result agrees well with the present experimental observations. A surface diffusion coefficient exceeding the bulk-liquid value has also been reported for thin methane films on a $\mathrm{MgO}$ substrate. ${ }^{12}$ The activation energies for self-diffusion in solid and liquid $\mathrm{Pb}$ are 1.11 and $0.19 \mathrm{eV}$, respectively. ${ }^{18,20}$ The intermediate value for the (110) surface of $0.65 \mathrm{eV}$ suggests that, over the temperature range covered in Fig. 3, surface diffusion is limited to some extent by the presence of residual crystalline order at the surface. ${ }^{10,12}$

The diffusional energy broadening, measured with $\mathrm{He}$ atom scattering, is of course characteristic only of those entities at the $\mathrm{Pb}(110)$ surface that contribute to the quasielastic signal. Most probably these are defects like adatoms, vacancies, or steps. Adatoms and vacancies are expected to be much more mobile than steps. ${ }^{21}$ Since the adatoms have a higher cross section for diffuse scattering than vacancies, ${ }^{22}$ we suggest that the intrinsic lateral diffusion coefficient determined here for the $\mathrm{Pb}(110)$ surface is actually that of $\mathrm{Pb}$ adatoms on the $\mathrm{Pb}(110)$ surface.

The data in Fig. 3 correlate nicely with results of a recent ion-scattering study from $\mathrm{Pb}(110){ }^{7}$ In this study the $\mathrm{Pb}(110)$ surface was found to become increasingly disordered at temperatures above $\simeq 450 \mathrm{~K}$. Up to $\simeq 580$ $\mathrm{K}$ a transition region is formed of about ten monolayers thickness, over which the order is gradually lost with distance from the underlying crystal to the surface. Above this temperature this region of transition moves into the bulk, leaving a surface which looks fully disordered in the ion-scattering measurements. Note that the disorder which is detected with ion scattering involves displace- 
ments of atoms away from lattice positions. Vacancies or adatoms on lattice sites remain undetected by this technique, in contrast to He scattering.

As all of the points in Fig. 3 have been obtained in a temperature range where both ion-scattering ${ }^{7}$ and $\mathrm{He}-$ diffraction measurements ${ }^{17}$ still detect residual lattice order at the surface, crystallinity effects are expected to play a role in the observed surface diffusion. For different diffusion models, different relations between $\Delta E$ and $\Delta \mathbf{K}$ are expected. The quadratic relation in Eq. (2) is valid only for random continuous diffusion, or, in the case of other diffusion models, only for small $\Delta K$ values (typically $<0.5 \AA^{-1}$ ). In the case of diffusion in a real liquid, minima are found in the energy width $\Delta E$ for $\Delta K$ values corresponding to maxima in the structure factor of the liquid. ${ }^{13}$ If the surface behaves like a twodimensional lattice fluid with jump diffusion over welldefined distances, the diffusional energy broadening should become a periodic function of $\Delta \mathbf{K} .^{23,24}$ Our analysis of the data in Fig. 3 in terms of Eq. (2) would underestimate the surface diffusion coefficient for each of these more complex types of diffusion. This leaves the value of $Q_{s}$ unaffected, but makes the above determined value of $D_{0}$ a lower estimate of the true preexponential factor.

Summarizing, we have shown that, with high energy resolution, He-atom scattering can be employed to study lateral-diffusion phenomena at surfaces. With this technique self-diffusion as well as diffusion of adsorbates can be investigated. This new method has been used here for the first time to detect liquidlike surface diffusivities at a melting $\mathrm{Pb}(110)$ surface. The activation energy for surface self-diffusion is found to be intermediate between the activation energies for self-diffusion in solid and liquid $\mathrm{Pb}$. Measurements of the precise relation between $\Delta E$ and $\Delta \mathbf{K}$ are in progress. From such measurements detailed information can be obtained concerning the microscopic diffusion dynamics and the pair-correlation function of the diffusing defects.

The authors gratefully acknowledge stimulating discussions with Professor J. R. Manson and a critical reading of the manuscript by Dr. B. J. Hinch. We thank A. J. Riemersma and A. C. Moleman of the University of Amsterdam for the preparation of our $\mathrm{Pb}$ specimen. One of the authors (J.W.M.F.) thanks the Alexander-
von-Humboldt Foundation for a fellowship.

${ }^{1}$ G. E. Bacon, Neutron Diffraction (Clarendon, Oxford, 1975).

${ }^{2}$ D. W. Bassett, in Surface Mobilities on Solid Materials, edited by $\mathrm{Vu}$ Thien Binh (Plenum, New York, 1983), pp. 63 and 83 .

${ }^{3}$ M. Tringides and R. Gomer, J. Chem. Phys. 84, 4049 (1986)

${ }^{4}$ H. P. Bonzel, in Ref. 2, p. 195, and references therein.

${ }^{5}$ G. E. Rhead, Surf. Sci. 47, 207 (1975).

${ }^{6} \mathrm{~J}$. F. van der Veen and J. W. M. Frenken, Surf. Sci. 178, $382(1986)$.

${ }^{7}$ J. W. M. Frenken, P. M. J. Marée, and J. F. van der Veen, Phys. Rev. B 34, 7506 (1986).

${ }^{8}$ B. Pluis, A. W. Denier van der Gon, J. W. M. Frenken, and J. F. van der Veen, Phys. Rev. Lett. 59, 2678 (1987).

${ }^{9}$ D.-M. Zhu and J. G. Dash, Phys. Rev. Lett. 57, 2959 (1986).

${ }^{10}$ V. Rosato, G. Cicotti, and V. Pontikis, Phys. Rev. B 33, 1860 (1986).

${ }^{11} \mathrm{~J}$. Q. Broughton and G. H. Gilmer, J. Chem. Phys. 79, 5119 (1983).

${ }^{12}$ M. Bienfait, Europhys. Lett. 4, 79 (1987).

${ }^{13}$ P. G. de Gennes, Physica (Utrecht) 25, 825 (1959).

${ }^{14}$ A. C. Levi, R. Spadacini, and G. E. Tommei, Surf. Sci. 121, 504 (1982).

${ }^{15}$ Our data should of course be described by the convolution of a Lorentzian energy profile with the (almost) Gaussian instrumental resolution function. Since, at present, we are only interested in the peak widths, the more convenient purely Gaussian description is sufficiently accurate for the modestly broadened peaks reported here.

${ }^{16} \mathrm{G}$. Armand, D. Gorse, J. Lapujoulade, and J. R. Manson, Europhys. Lett. 3, 1113 (1987).

${ }^{17}$ J. W. M. Frenken, J. P. Toennies, and Ch. Wöll, to be published.

${ }^{18}$ N. H. Nachtrieb, Ber. Bunsen-Ges. Phys. Chem. 80, 678 (1976).

${ }^{19}$ J. D. Wrigley and G. Ehrlich, Phys. Rev. Lett. 44, 661 (1979).

${ }^{20}$ J. W. Miller, Phys. Rev. 181, 1095 (1969).

${ }^{21}$ A. Lahee, J. R. Manson, J. P. Toennies, and Ch. Wöll, Phys. Rev. Lett. 57, 471 (1986).

${ }^{22}$ E. Zaremba, Surf. Sci. 151, 91 (1985).

${ }^{23}$ C. T. Chudley and R. J. Elliot, Proc. Phys. Soc. London 77, 353 (1961).

${ }^{24}$ M. Bienfait, J. P. Coulomb, and J. P. Palmari, Surf. Sci. 182, 557 (1987). 